\title{
Crossover from thermal to quantum creep in layered antiferromagnetic superconductor
}

\author{
Tomasz Krzysztoń. \\ Institute for Low Temperatures and Structure Research, Polish Academy of \\ Sciences, 50-950 Wroctaw, Poland.
}

\begin{abstract}
The influence of the antiferromagnetic order on the superconductor in the mixed state results in creation of spin-flop domains along the cores of the vortex lines. It is shown that this phenomenon makes possible crossover from quantum creep regime to thermal one, and vice versa, at constant temperature. To do this one needs to simply change the intensity or the direction of applied magnetic field in the basal ab plane of layered structure.
\end{abstract}

Key words: superconductivity; magnetic superconductors; mixed state; quantum creep

PACS: :7430C;7430E;7460G;7470H;

\section{Introduction}

The suppression of superconductivity by applied magnetic fields implies that magnetism and superconductivity are two very different cooperative phenomena that are unlikely to be present simultaneously within the same sample. On the other hand, there exist materials in which both competitive phenomena do coexist in the same volume. This happens because the $4 \mathrm{f}$ electrons of rare earth $(R E)$ ions, in most cases responsible for magnetism, and those electrons responsible for superconductivity are spatially well-separated in regular crystal lattice[1,2]. The specific feature caused by the long-range antiferromagnetic order in the mixed state of superconductor is the creation of spinflop (or metamagnetic) domain along each vortex core [3]-[6]. This is easy to understand by taking two sublattices antiferromagnet as an example. An infinitesimal magnetic field applied perpendicular to the easy axis makes the ground antiferromagnetic state unstable against the phase transformation to the canted phase (spin-flop). On the contrary, if the magnetic field is applied parallel to the easy axis the antiferromagnetic configuration is stable up to 
the thermodynamic critical field $H_{T}$. When the field is further increased a canted phase develops in the system. Assume that in the antiferromagnetic superconductor the lower critical field fulfils the relation $H_{c 1}<\frac{1}{2} H_{T}$ and that the external field, $H_{c 1}<H<\frac{1}{2} H_{T}$, is applied parallel to the easy axis. Then the superconducting vortices appear in the ground antiferromagnetic state. What happens if the field is increased above $\frac{1}{2} H_{T}$ ? The phase transition to the canted phase originates in the vortex core because the field intensity in the core doubles the external one. The spatial distribution of the field around the vortex is a decreasing function of the distance from its center. Hence the magnetic field intensity in the neighborhood of the core is less then $H_{T}$. Therefore, the rest of the vortex remains in the antiferromagnetic configuration. The radius of spin flop domain grows as the field is increased. There exists critical field for flux penetration in the form of vortices with canted phase along the core $[3,4]$. When the external field is applied perpendicular to the easy axis, the vortices do not change their structure and behave quite similarly to the ones in nonmagnetic superconductor. Thus, in the considered model there are two distinct types of vortices. The above considerations apply to classical superconducting Chevrel phases as well as to the high $T_{c}$ superconductors, where antiferromagnetic order is produced by the regular lattice of rare earth $(R E)$ ions occupying isolating layers. A typical example of such system is $\mathrm{ErBa}_{2} \mathrm{Cu}_{3} \mathrm{O}_{7}$. This compound has tetragonal unit cell with small orthorombic distortion in the $a b$ plane. The $E r$ ions form two sublattices antiferromagnetic structure of magnetic moments laying parallel and antiparallel to the $\mathbf{b}$ direction [7]. Another example may be $R E$ nickel boride- carbides [8]. Their structure consists of $R E$-carbon layers separated by $N i_{2} B_{2}$ sheets. The theoretical structure used in the calculations in the present model is shown in Fig.1. It consists of superconducting layers of thickness $d_{s}$ and isolating ones of thickness $d_{i}, d=d_{i}+d_{s}$. In the isolating layers, the magnetic moments are running parallel and antiparallel to the $\mathbf{b}$ direction (easy axis). The magnetic field aligned parallel to the conducting planes makes the vortex lattice to accommodate itself to the layer structure so that the vortex cores lie in between the superconducting sheets. A current density $j$, flowing along the planes perpendicular to the applied magnetic field exerts a Lorentz force on the vortices in the $\mathbf{c}$ direction so that intrinsic pinning barriers are formed on strongly superconducting layers. This paper is devoted to the problem of resistive properties of the system caused by the quantum creep. In the previous paper [6] thermally assisted flux motion was considered. The experimental evidence of quantum tunneling is based on the fact that the magnetic moment relaxation rate exhibits two types of behavior as a function of temperature. Above a characteristic temperature $T_{0}$ in the thermal activation regime the decay rate is of the Arrhenius type $\Gamma \sim \exp \left(-U_{0} / k_{B} T\right)$. Below $T_{0}$, the decay rate is essentially independent of temperature $\Gamma \sim \exp (-S / \hbar)$ and is interpreted as arising from the quantum tunneling of vortices through intrinsic pinning potential $[9,10]$. The purpose of the present calculations is to show drastic change of tunneling rate and crossover temperature due to the phase 
transition to the canted phase around the vortex core.

\section{CALCULATION OF CROSSOVER TEMPERATURE}

Consider the vortex line as a straight string-like object of an effective mass $M$ per unit length trapped into a metastable state in an intrinsic pinning potential $V(u)$ and exposed to continuous deformation $u(x, t)$ in the $\mathbf{z}$ direction. The magnetic field is applied in $\mathbf{x}$ direction (b direction on Fig.1). In the semiclassical approximation the quantum decay rate is calculated as a saddle-point solution (bounce) of the Euclidean action $S$ for the string

$$
\begin{aligned}
& S=\int_{-\infty}^{\infty} d x \int_{0}^{\hbar \beta}\left\{d \tau \frac{1}{2} M\left(\frac{\partial u}{\partial \tau}\right)^{2}+\frac{\varepsilon_{l}}{2}\left(\frac{\partial u}{\partial x}\right)^{2}+V(u)\right\} \\
& -\int_{-\infty}^{\infty} d x \int_{0}^{\hbar \beta}\left\{\frac{\eta}{2 \pi} \frac{\partial u}{\partial \tau} \int_{0}^{\hbar \beta} d \tau^{\prime} \frac{\partial u}{\partial \tau^{\prime}} \ln \left|\sin \frac{\pi}{\hbar \beta}\left(\tau-\tau^{\prime}\right)\right|\right\} .
\end{aligned}
$$

Here $\beta=\left(k_{B} T\right)^{-1}, \varepsilon_{l}$ is the line tension of the vortex, $\eta$ is the viscosity coefficient and $\tau$ denotes imaginary time. The pinning potential $V(u)$ consists of intrinsic periodic part and the Lorentz potential:

$$
V(u)=-\frac{\varphi_{0} j_{c} d}{2 \pi} \cos \left(\frac{2 \pi u}{d}\right)-\varphi_{0} j u
$$

For large current, this potential can be expanded around the inflection point to give

$$
V(u)=V_{0}\left[\left(\frac{u}{w}\right)^{2}-\left(\frac{u}{w}\right)^{3}\right]
$$

where $V_{0}=\frac{2}{3} \frac{\varphi_{0} j_{c}^{2} \pi^{2}}{d^{2}} w^{3}$ and $w=\frac{3 d}{\pi}\left(\frac{j_{c}-j}{2 j_{c}}\right)^{\frac{1}{2}}$ may be thought as the width of the barrier because $V(0)=V(w)=0, j_{c}$ is the critical depinning current. The last term in Eq.(1) is the so-called Caldeira-Leggett action [11], which describes ohmic damping produced by the coupling to the heat-bath of harmonic oscillators. The line tension $\varepsilon_{l}$ is different for vortices in two different orientations in the ab plane. As discussed above the vortices lying parallel to a direction and those laying in the $\mathbf{b}$ direction but created in the magnetic field fulfilling relation $H_{c 1}<H<\frac{1}{2} H_{T} \quad$ [6] have the line tension equal to

$$
\varepsilon_{l}=\varepsilon_{a}=\varepsilon_{0} \ln \frac{\lambda_{a b}}{d}
$$


where $\varepsilon_{0}=\frac{\varphi_{0}^{2}}{16 \pi^{2} \lambda_{a b}^{2} \mu_{0}}$. For those vortices lying in the $\mathbf{b}$ direction but possessing spin flop domain, we write the following expression [5]

$$
\varepsilon_{l}=\varepsilon_{b}=\frac{\varphi_{0} H_{T}}{2}+\frac{9}{128} \varepsilon_{0} \ln \frac{\kappa^{2} \varphi_{0}}{\pi \lambda_{j}^{2} B_{T}} .
$$

Here $B_{T}=H_{T}+2 M_{0}, 2 M_{0}$ is the magnetization of the spin flop domain, $\lambda_{j}=$ $\frac{\lambda_{c}}{\lambda_{a b}} d, \lambda$ denotes penetration depth of the magnetic field and $\kappa$ is the GinzburgLandau parameter in the ab plane. In the semiclassical approximation the decay rate is given by the value of the action on a classical trajectory obtained from the Euler-Lagrange equations of the motion

$$
-M \frac{\partial^{2} u}{\partial \tau^{2}}-\varepsilon_{l} \frac{\partial^{2} u}{\partial x^{2}}+V^{\prime}(u)+\frac{\eta}{\hbar \beta} \int_{0}^{\hbar \beta} d \tau \frac{\partial u}{\partial \tau} \cot \frac{\pi}{\hbar \beta}\left(\tau-\tau^{\prime}\right)=0 .
$$

The trajectory $u_{0}(x)$ for static solution of Eq.(5) gives the activation energy in the thermal regime $T>T_{0}$. Below this crossover temperature a new kind of trajectory, periodic in imaginary time, develops. Therefore, $u(x, \tau)$ can be expanded in the Fourier series with Matsubara frequencies

$$
u(x, \tau)=\sum_{n=0}^{\infty} u_{n}(x) \cos \left(\omega_{n} \tau\right) \quad ; \quad \omega_{n}=\frac{2 \pi n}{\hbar \beta} .
$$

Substituting this expansion into Eq.(5) and linearizing potential around the static solution $u_{0}(x)$ one obtains

$$
-\varepsilon_{l} \frac{\partial^{2} u_{n}}{\partial x^{2}}+V^{\prime \prime}\left(u_{0}\right) u_{n}=-\left(\eta \omega_{n}-M \omega_{n}^{2}\right) u_{n}
$$

Upon introducing new variables $v_{n}=\frac{u_{n}}{w}$ and $\zeta=\frac{x}{d}\left(\frac{\pi^{2} w \varphi_{0} j_{c}}{\varepsilon_{l}}\right)^{\frac{1}{2}}$ the static equation now reads.

$$
-\frac{1}{2} \frac{\partial^{2} v_{0}}{\partial \zeta^{2}}+2 v_{0}-3 v_{0}^{2}=0
$$

Its solution is easily found to be

$$
v_{0}=\cosh ^{-2} \zeta
$$

Substitution Eq.(8) into Eq.(7) results in the following equation

$$
-\frac{1}{2} \frac{\partial^{2} v_{n}}{\partial \zeta^{2}}+2\left(1-3 \cosh ^{-2} \zeta\right) v_{n}=E_{n} v_{n}
$$

where

$$
E_{n}=-\frac{j_{c} w^{2}}{V_{0}^{2}}\left(\eta \omega_{n}+M \omega_{n}^{2}\right)
$$


Eq.(10) has three discrete eigenvalues -5/2,0,3/2 [12]. The negative one determines the crossover temperature

$$
k_{B} T_{0}=\frac{\hbar \eta}{4 \pi M}\left\{\left[1+\frac{20 \pi \varphi_{0} j_{c} M}{d \eta^{2}}\left(\frac{j_{c}-j}{2 j_{c}}\right)^{\frac{1}{2}}\right]^{\frac{1}{2}}-1\right\}
$$

\section{ESTIMATION OF EFFECTIVE MASS AND VISCOSITY}

The above calculations apply to both kinds of vortices. The only difference is their effective mass and viscosity coefficient. It is possible to express these parameters as the function of condensation energy accumulated in the vortex cores. For the stationary flux flow at $j>j_{c}$ the viscous force $\eta \frac{\partial u}{\partial t}$ is equal to Lorentz force. The electric field generated by the moving vortex is $E=B \frac{\partial u}{\partial t}$, so we get $E=\frac{\varphi_{0} B}{\eta} j=\rho j=\rho_{N} \frac{B}{H_{c 2}} j$ where $\rho_{N}$ is the normal phase resistivity in the $a b$ plane and $H_{c 2}$ is the upper critical field parallel to the layers. Finally,

$$
\eta=\frac{\varphi_{0} H_{c 2}}{\rho_{N}}=\frac{\varphi_{0} \kappa H_{c} \sqrt{2}}{\rho_{N}}=\varepsilon_{l} \frac{4 \sqrt{3} \kappa^{2}}{\pi \rho_{N} \ln \kappa},
$$

where $H_{c}=\frac{\varepsilon_{l} \kappa 2 \sqrt{6}}{\pi \varphi_{0} \ln \kappa}$ is calculated from the constitutive relation $\varepsilon_{l}=H_{c 1} \varphi_{0}$. The effective mass of the vortex can be deduced from the work of Suhl [13]. He derived the core contribution to the inertial mass $m_{\text {core }}=\frac{3}{8} m_{e} \frac{\xi^{2} H_{c}^{2} \mu_{0}}{\epsilon_{F}}$, where $m_{e}$ denotes the mass of the electron and $\epsilon_{F}$ is the Fermi energy, and the electromagnetic contribution coming from the energy of the electric field induced by the moving flux. Simple estimation shows that this contribution in layered superconductors is $10^{-4}$ of the core contribution. Therefore,

$$
M=\varepsilon_{l}^{2} \frac{9 \lambda_{a b}^{2} m_{e} \mu_{0}}{\varphi_{0}^{2} \pi^{2} \epsilon_{F}(\ln \kappa)^{2}} .
$$

It is possible now to relate the crossover temperature in Eq.(11) to the line tension of the vortex

$$
T_{0}=\alpha \varepsilon_{l}^{-1}
$$

The coefficient $\alpha$ depends on the material constants and current intensity $j$.

\section{CROSSOVER FROM QUANTUM TO THERMAL CREEP}

As was mentioned in the introduction there are two tapes of vortex lines in the system. The first ones, without magnetic domain, occur when the field 
is applied in the $\mathbf{a}$ or $\mathbf{b}$ directions, but its intensity does not exceed $\frac{1}{2} H_{T}$. Eq.(3) gives their line tension and the related crossover temperature is given by $T_{0 a}=\alpha \varepsilon_{a}^{-1}$. The other type, possessing magnetic domain, occurs when the field is applied in the $\mathbf{b}$ direction and its intensity exceeds $\frac{1}{2} H_{T}$. Eq. (4) gives their line tension and the crossover temperature is $T_{0 b}=\alpha \varepsilon_{b}^{-1}$. It is easy to see that $\varepsilon_{b}>\varepsilon_{a}$ and therefore $T_{0 a}>T_{0 b}$. The above calculations lead to the following conclusion. It is possible to switch the creep regime at constant temperature. To do this, one needs to change the field intensity or simply change the field direction in the ab plane. The diagrams in Fig.2 show possible scenarios of crossover from quantum to thermal regimes. Let us discuss just two of these scenarios. Here, the first prescription is as follows. Fix the temperature $T_{0}$ somewhere in the range $T_{0 a}>T_{0}>T_{0 b}$. Then align the external field in the a direction and increase its intensity to the point marked II on the upper diagram in Fig.2. The system is in the quantum creep regime now. Then move the direction of external field from $\mathbf{a}$ to $\mathbf{b}$ axis. The system jumps to the point II of the lower diagram and finds itself in the thermal creep regime. Doing the same operations in the reverse order one enforces the system to crossover from thermal to quantum creep regime. The other scenario is the following. Apply magnetic field along $\mathbf{b}$ axis and increase its intensity above $\frac{1}{2} H_{T}$ keeping temperature constant in the interval. $T_{0 a}>T_{0}>T_{0 b}$. Now, the system switches from quantum to thermal regime.In other words, the system moves along the line defined by points I and II on the lower diagram of Fig.2.

\section{ACKNOWLEDGEMENTS}

This work was supported by Komitet Badań Naukowych under grant 2 PO3B 12519

\section{References}

[1] O.Fischer, M.B.Maple (Eds.), Superconductivity in Ternary Compounds, Springer,Berlin, 1983.

[2] J.W.Lynn, J.Alloys and Compounds 181 (1992) 419

[3] T. Krzysztoń, J. Magn. Magn. Materials, 15-18, 1572 ,(1980)

[4] T. Krzysztoń, Phys. Lett., 104A, 225, (1984)

[5] T. Krzysztoń, Phys. Lett., A190, 196, (1994)

[6] T. Krzysztoń, Physica C 294, 47, .(1998). 
[7] J.Zaretsky, C.Stassis, A. I. Goldman, P. C. Canfield, P. Dervenagas, B. K. Cho and D. C. Johnston, Phys. Rev., B51, 678. (1995)

[8] S. K. Sinha, J. W. Lynn, T. E. Grigereit, Z. Hossain, L. C. Gupta, R. Nagarajan, C. Godard, Phys. Rev., B51, 681, (1995)

[9] B.I.Ivlev, Yu.N.Ovchinnikov and R.S.Thompson, Phys. Rev, B44, 7023, (1991).

[10] W.M.Gaber and B.N.N.Achar, Phys. Rev, B52, 1314, (1995)

[11] A.O.Caldeira and A.J.Leggett, Ann.Phys (N.Y), 149, 374, (1965)

[12] L.D.Landau, E.M.Lifshitz, Quantum Mechanics, Oxford, Pergamon Press 1962

[13] H.Suhl, Phys.Rev.Lett, 14, 226, (1965)

\section{FIGURE CAPTIONS}

Fig.1. Schematic drawing of a layered antiferromagnetic superconductor. The rare earth elements form two sublattices of magnetic moments (bold arrows) lying parallel and antiparallel to $b$ direction. The reference frame and the crystallographic axes are shown.

Fig.2. Schematic diagrams showing possible ways ( marked by arrows ) of changing quantum creep behavior in the system to thermal one, and vice versa. Shaded areas on the diagrams correspond to quantum creep regime. 
This figure "Fig1.jpg" is available in "jpg" format from: http://arxiv.org/ps/cond-mat/0011482v1 
This figure "Fig2.jpg" is available in "jpg" format from: http://arxiv.org/ps/cond-mat/0011482v1 